Scientific Electronic Archives

Issue ID: Sci. Elec. Arch. Vol. 15 (2)

February 2022

DOI: http://dx.doi.org/10.36560/15220221507

Article link: https://sea.ufr.edu.br/SEA/article/view/1507

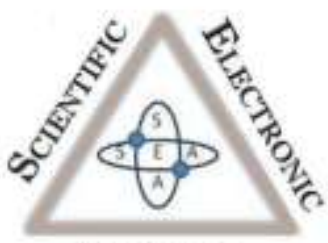

Archives

ISSN 2316-9281

\title{
Mobile Applications for stress management
}

\author{
Marina Anagnostou \\ N.C.S.R. "Demokritos", Greece \\ Corresponding author \\ Athanasios Drigas \\ N.C.S.R. Demokritos, Greece \\ dr@iit.demokritos.gr
}

\begin{abstract}
Stress continues to be one of the challenging of responding in different situations. It evokes negative thoughts and feelings in a person. It is known that there are many ways to reduce stress, like counseling, training, exercise, e.t.c. Although, nowadays, through technology have developed different ways to face with it. There are many applications in smartphones and tablets, which are designed for stress management. These applications contain many and different techniques to reduce stress, such as meditation, mindfulness breathing, cognitive behavior therapy and relaxation techniques.
\end{abstract}

Keywords: stress management, mobile applications, intervention, mental health

\section{Introduction}

Nowadays, stress is a growing problem, which affecting many people all over the world and in the most times has negative results (Carissoli, C.et.al. 2015). According to the world Health Organization mental health problem and stressrelated disorders are the biggest overall cause of early death in Europe and for that reason it is important people learn how to deal with it.[2] In recent years, research concerned with technology and how it can help in stress reduction and as a result, many mental health applications have been developed for stress management (Carissoli, C.et.al. 2015).

Stress management is a wide spectrum of techniques aimed at controlling a person's level of stress, especially chronic stress, usually for the purpose of improving everyday functioning. In this article we will focus on to some of them mobile applications that exist and what their contribution is on stress management. There are two kinds of applications: these one, which use mainly text instructions for relaxation training and breathing exercises and the others, which monitoring and recording emotional status by simple and easy text questions that appear on the screen of the mobile device.( Gaggioli, A et. A;. 2014).

In addition, mobile phone technologies have been hailed as a promising means in mental health interventions. Smartphones, for example, are the new generation technology, which have features for mental health and are expanding with technology advancements and include larger screens, easier data input and lighter weight optimizes userfriendliness. (Seko, Y et. Al., 2014, Callan, J , 2017), while they are commonly used in education( $Z$ Karabatzaki et. al., 2018) and in special Education in order to help students and promote inclusion (A Stathopoulou et. al., 2019).

Furthermore, researches have shown that mobile applications are perceived as useful and can provide helpful information regarding to mental health problems. Also stress is connected with Metacognition (A Drigas, E Mitsea, 2021) and there are mobile applications which help people with stress by initiating various kind of feedback including visual feedback and auditory feedback. (Becker, B. 2016, Dr. Hempel, 2018).

In conclusion, mobile phone technology is growing in leaps and bounds, result in frequent app updates and thus the change of features and functions. This prospect will be able to evolve stress management by mobile applications and help many people in future, who suffer from stress(Wang, $\mathrm{K}$ et al., 2018).

\section{Backround \\ Stress management}


Stress is a body reaction to any change that requires an adjustment or response. The body reacts to these changes with physical, mental and emotional responses. High levels of stress can have negative effects on people and it is strongly associated with mental health problems, such as depression. (Rizzolo et. Al., 2018).

Thus, when stress when not dealt with, effectively can cause anxiety, but when it is in low levels can motivate people to perform well with it and preserve their mental and physical well being(Galante, J, 2018). There are different types of stress, one of these is chronic stress and that is which has become increasingly important public health concern(Gaggioli, A, 2014). Chronic stress presents a growing pervasive burden in health care, but mobile applications have the potential to deliver stress management strategies. The most common strategies are mindfulness and meditation (Coulon, M. et. Al. 2016).

Also, deep and slow breathing exercises can be effective in the treatment of stress and recently mobile applications have been proposed as novel breathing training tools. In this case, smartphones can be a novel opportunity to improve breathing training, because they follow their users anywhere. So, a breathing training application could be always available to support users at any moment (Chittaro, L., Sioni, R., 2014).

Although, stress is a growing problem affecting many people, it is important to learn how to deal with it Carissoli, C.et.al. 2015). In this case, prevention and management of work-related stress and related mental problems are a great challenge and mobile applications are a promise to integrate prevention strategies too (Ahtinen, A et. Al., 2013).

In conclusion, we can observe through researches that using naturally calming elements in mobile applications and providing positive feedback on users actions then can minimize stressors for users(Ahtinen, A et. Al., 2013).

\section{Mobile Application}

Mobile applications for mental health have a potential to be effective in monitoring or improving symptoms of certain mental disorders, such as anxiety, stress, depression, e.t.c. They are showing promise to ease of use and access, while educating users on self-management. (Wang, $\mathrm{K}$ et. Al. , 2018, Martín, B et. Al., 2018). Mobile health offers an extraordinarily powerful platform for monitoring and management of mental health symptoms and disorders. It uses the function of mobile devices, but most relies on the download of mobile applications, which are being used increasingly for mental health purposes. There is a research, which has shown that there are 32 stress management applications in IOS app store, which reduce the burdens associated with chronic stress (Coulon, M et. Al., 2016).

Christmann, A. C.(2017), et. al., in their article are mentioned to these applications, which were available through Google Play in October 2015. These applications were identified using the search terms "stress management," "stress reduction," and "stress relief". For each search term, the first 250 free apps were checked with some criteria such as: Apps had to be provided in the "Health \& Fitness" or "Medical" categories of Google Play to exclude apps that focus on "Entertainment" , "Beauty," or "Music \& Audio." In this study were chosen applications only for stress management and each app allowed the users to progress at their own speed, allowing both raters to thoroughly check all features of the apps until it was apparent that no new features were going to be activated. The results showed that many of these applications were effective in stress management, although some of these apps only provided information about stress without any further advice on how to cope with it.

In this review will be mentioned to 11 mobile applications, which were designed to help reducing stress(Coulon, M et. Al., 2016).

Carissoli, C. et.al (2015), in their article referred to a first study in the field showing that an application can simplify the mindfulness approach making it usable in daily life. This app, It's time to relax, was created by using the Eclipse Integrated Development Environment (IDE). The implementation was tested on a Samsung Galaxy $Y$ terminal, kindly loaned by Samsung Italia, equipped with Android v2.2. To verify the changes in perceived stress level, the Italian validated version of the Mesure du Stress Psychologique (MSP) questionnaire was used, which evaluates one's perceived stress level within the preceding 3 months. The Cronbach's alpha of the Italian validated version is 0.95 . It includes 49 items and considers six dimensions: (a) loss of control and irritability, (b) physiological feelings, (c) sense of effort and confusion, (d) depressive anxiety, (e) pain and physical problems, and (f) hyperactivity and accelerated behaviors. The MSP was administered before and 3 weeks after the interventions. This study shows that both music and meditation were perceived as undemanding, and participants felt that they had learned useful strategies (Carissoli, C.et.al. 2015).

Subsequently, Gaggioli, A. et.al (2014) referred to a free mobile application, Positive Technology, which is for the self-management of psychological stress. "The Positive Technology application consists of the following three main components:

a) Guided Relaxation, b) Biofeedback, c) Stress self-reporting and tracking. The findings of this study show that the relaxation exercises performed with the application were effective in reducing stress, as indicated by the significant decrease in self-reported levels of anxiety and by the increase of positive values after the relaxation session. Positive Technology is the first free mobile stress management platform available on the market which combines relaxation training, biofeedback and interactive 3D contents.

Moreover, Coulon, M. S. , Monroe, M. C. , West, D. S. (2016) in their review, which is the first 
systematic review of apps for stress management and the first review to provide guidance on evidence-based, transparent, and functional and user-friendly apps, referred that chronic stress presents a growing, pervasive burden in healthcare, and mobile applications have the potential to deliver evidence-based stress management strategies. One of these applications, which has mentioned in their article, is Breath2Relax. «Breath2Relax, which is for diaphragmatic breathing, was recently demonstrated as cost effective for stress reduction within the Department of Veterans Affairs, with estimates that once at least 1,600 individuals have used the app, it saves $\$ 1$ million annually relative to standard care, Breathe2Relax had been used by 47,000 patients at the time of publication and it provides a strong example of a well-developed app that excels across all domains and demonstrates the potential of apps to improve health on a scalable level. »

In addition, Baker, D. , Rickard, N. (2018) examined the effectiveness of a self-monitoring mobile phone application by investigating the relationships between app engagement and mental health outcomes. This is application is MoodPrism. «MoodPrism is an iOS and Android app, freely available on the Australian app stores, designed to: a) help users track their moods over time; and b) collect data from users regarding their emotional states over time in natural everyday contexts (Rickard et al., 2016). After completing a set of surveys to obtain baseline measurements, users are prompted at a random time throughout their day to complete a daily mood survey, which asks them 12 questions about how they are feeling, where they are, and what they have been doing. This information is formatted in a mood diary, which users can review to obtain feedback about how they have been feeling over time and in what contexts. After 30 days users are prompted to complete a second set of follow-up surveys, enabling collection of data to evaluate the effectiveness of the app. » The results from this app were positive, as MoodPrism predicted improvements in mental health (depression and anxiety) and wellbeing. The primary finding was that app engagement ratings on the application predicted changes in depression, anxiety, and mental wellbeing ratings. This indicates that, in general, participants who had more positive and engaging experiences using MoodPrism experienced greater decreases in depression, greater decreases in anxiety, and greater increases in mental wellbeing than those who did not rate their engagement as highly.

Chittaro, L. \& Sioni, R. (2014) are mentioned to breathing techniques for stress management and developed three versions of a mobile app for the Android platform that differ only in the way instructions are presented. «The app guides users in practicing deep and slow circular breathing: a breathing cycle is composed of two phases (inhalation and exhalation) of the same length and the breathing frequency is 6 cycles per minute. First version is Voice only, which provides instructions through audio only. In particular, employ the same voice instructions of Tactical Breather, "inhale", "2", " 3 ", and "4" for the inhalation phase, and "exhale", "2", "3", and "4" for the exhalation phase. The time between any two consecutive instructions is set at $1.25 \mathrm{~s}$. Second version is Sphere, which was implemented to replicate the visualization used for inhalation and exhalation in Tactical Breather. It employs a green inflating sphere to instruct users to inhale, and a red deflating sphere to instruct them to exhale. Third version is Wave, which employs a moving green triangle wave to instruct users about when to inhale (the wave is rising) and exhale (the wave is falling), similarly to existing apps like VitalEQ Respiroguide and Paced Breathing.».

One more application is the Get Happy, which is available in mobile phones and is created for people who suffer from depression. After 3 months research, the results showed that people who used it, had significant benefits in depression reduction. [4]

Moreover, Tyszkiewicz, F. M. et.al (2017) made a research for a Stressless intervention, which is an iOS app-based intervention designed to reduce stress and improve wellbeing. This program includes five modules and people, who use it are encouraged to work through each module in sequence, and at their own pace. Each module comprises audio-based activities and presentations, and each of these has transcripts available for individuals who would rather read or prefer text for accessibility reasons. There are also selfcheck and reflective activities to help establish key strategies and practice and reinforce the skills covered within the intervention program to deal more effectively with daily stressors. In addition to this modularized content, participants also have access to a monitoring component, involving a self-report survey feature. This allows them to enter their current stress levels and mood, as well as key contextual variables, such as the specific coping strategies used in response to specific stressful events, and their confidence at managing these stressors. These stress responses are graphed and fed back to participants so that they can both gain insight into their stress and mood in daily life and also chart progress and improvement in these symptoms as they continue to use the intervention. This article analyzes in detail all the steps of using this program and the results showed that findings of improved psychological functioning amongst the group of Stressless app users as well as findings suggesting that participants found the app easy and intuitive to use suggests that Stressless may be a valuable resource for people experiencing high levels of distress.

Ahtinen A. et.al,(2013) in their article referred to a study, which was to study the usage, acceptance, and usefulness of a mobile mental wellness training application among working-age individuals, and to derive preliminary design implications for mobile apps for stress management. For this, was designed a mobile app, called Oiva, 
which based on acceptance and commitment therapy and was for supporting active learning of skills related to mental wellness through exercises in the daily life. This study showed that this app was easy to use and acceptable from the participants. It had significant improvement and the active usage, observed positive effects on wellness, and the generally positive user experiences of Oiva suggest that it is possible to develop engaging mobile apps that are experienced as beneficial for personal mental wellness.

Reid SC, et.al, (2011) referred to a program, which trailed in primary care to examine the mental health benefits to it, called Mobiletype. Mobiletype program is a mental health assessment and management mobile phone application which monitors mood, stress, coping strategies, activities, eating, sleeping, exercise patterns, and alcohol and cannabis use at least daily. The results of this study suggested that participation in this program lead to enhanced mental health care at pre-test and improved mental health outcomes for the sample as a whole.

Morrison G. L., et.al (2018) in their review, are mentioned in a application, called Healthy mind. Healthy mind is an app intervention that offers a range of evidence-based tools for managing emotional distress. In this review a comparison is made between a web-intervention and an appintervention and the results showed that the usage of a web-based stress management intervention differed from usage of an app-based intervention that shared the same underlying theoretical components. Web users logged in fewer times for a longer period of time whereas app users logged in more frequently for shorter periods of time. This usage didn't give conclusions for the effective levels, so a future study needed.

Finally, mobile well being apps represent a promising approach to encourage adherence to a healthy lifestyle anywhere and at any time. Therefore, the expectation that mobile applications are easy and intuitive to use might indirectly influence the perception of mobile mental health treatment.

\section{Interventions and Stress Management}

There are too many interventions using real time mobile technology, which improve health outcomes in several domains, including stress management. Intervention research on stress management has mostly been carried out in occupational settings and at schools. For the present, the focus is on adult populations in occupational or community settings. In the ideal world, stress management skills would be trained already in early childhood to develop healthy coping strategies that can prevent stress-related problems in later life. In real life, this does not usually happen and, hence, adults struggling with stress need supportive interventions (Kaipainen, K. , 2014).

Mobile interventions, which used to help to coping with stress include relaxation training, breathing techniques, music, cyber intervention based on inoculation training methodology. Furthermore, there are interventions based on cognitive behavioral techniques, which has been proven that are very effective in stress management. (Ahtinen, A et. Al., 2013).

Grossmann, P., et. Al (2003)., referred to a Mindfulness-based stress reduction and they mentioned that MBSR as an intervention is usefulness for a broad range of a chronic stress. Mindfulness training may be an intervention with potential for helping many to learn to deal with chronic disease and stress. Nevertheless, we now need to test these claims more thoroughly by using well-defined patient populations, applying more stringent methodological procedures, and assessing objective disease markers in addition to selfreported psychosocial and functional indicators of distress.

Finally, mobile phones facilitate integrating interventions in daily lives of people, which allow monitoring their activities and their contexts. There are studies, which indicate that mobile phones are potentially effective platforms for enhancing treatment and improving mental health interventions among people (Seko, Y et. Al. 2014).

\section{Conclusion}

Stress management is ongoing struggle for many people in the developed world. The consequences of chronic stress are born at both individual and social level. Technology offers potential solutions for daily self-management. It is expected that the mobile applications related to management and control of stress will evolve in the coming years adding new functionalities until they become fully integrated self-management systems, and more and more apps are likely to include the above mentioned professional assistance services and online support communities (Martín, B et. Al., 2018). In conclusion, we could mention that technology is the future, but should be seen as a tool to assist in the change process (Ahtinen, A et. Al., 2013).

\section{References}

Carissoli, C., Villani, D., Riva, G. (2015). Does a Meditation Protocol Supported by a Mobile Application Help People Reduce Stress? Suggestions from a Controlled Pragmatic Trial. Published in: CYBERPSYCHOLOGY, BEHAVIOR, AND SOCIAL NETWORKING, Volume 18, Number 1, DOI: 10.1089/cyber.2014.0062. Retrieved from: www.researchgate.net

Gaggioli, A., Cipresso, P., Serino, S., Campanaro, D.M., Pallavicini, F., Wiederhold, B. \& Riva, G. (2014). Positive Technology: A Free Mobile Platform for the SelfManagement of Psychological Stress. Annual Review of Cyber therapy and Telemedicine 2014 v B.K. Wiederhold and G. Riva (Eds.), 33-37 Retrieved from: www.researchgate.net

Seko, Y., Kidd, S., Wiljer, D., McKenzie, K. (2014). Youth mental health interventions via mobile phones: a scoping 
review. Published in: Cyberpsychol Behav Soc Netw. 2014 Sep;17(9):591-602. doi: 10.1089/cyber.2014.007. Retrieved from: www.ncbi.nlm.nih.gov

Callan, J. A., Wright, J., Siegle, J. G., Howland, H. R., Kepler, B. B. (2017). Use of Computer and Mobile Technologies in the Treatment of Depression. Published in: Archives of Psychiatric Nursing, Volume 31, Issue 3 , June 2017, doi: 10.1016/j.apnu.2016.10.002, 311-318 Retrieved from: www.sciencedirect.com

Drigas, A., \& Mitsea, E. (2021). Metacognition, StressRelaxation Balance \& Related Hormones. Int. J. Recent Contributions Eng. Sci. IT, 9(1), 4-16.

Stathopoulou, A., Karabatzaki, Z., Tsiros, D., Katsantoni, S., \& Drigas, A. (2019). Mobile apps the educational solution for autistic students in secondary education.

Karabatzaki, Z., Stathopoulou, A., Kokkalia, G., Dimitriou, E., Loukeri, P. I., Economou, A., \& Drigas, A. (2018). Mobile Application Tools for Students in Secondary Education. An Evaluation Study. International Journal of Interactive Mobile Technologies, 12(2).

Becker, B. (2016). Acceptance of mobile mental health treatment applications. The 6th International Conference on Current and Future Trends of Information and Communication Technologies in Healthcare. 220-227. Available online at www.sciencedirect.com

(2018). How effective are digital apps in meditation \& stress reduction? SCIENCE SERVICE, Dr. Hempel Digital Health Network. Retrieved from: www.dr-hempelnetwork.com

Wang, K., Varma, D. S., Prosperi, M. (2018). A systematic review of the effectiveness of mobile apps for monitoring and management of mental health symptoms or disorders. Published in: J Psychiatr Res. 2018 Dec;107:73-78. doi 10.1016/j.jpsychires.2018.10.006. Retrieved from: www.ncbi.nlm.nih.gov

Rizzolo, Simpkins, S., Zipp, P. Z., Stiskal, D. (2009). Stress Management Strategies For Students: The Immediate Effects Of Yoga, Humor, And Reading On Stress. Avalaible in: Journal of College Teaching and Learning 6(8):79-88 . January 2009, DOI: 10.19030/tlc.v6i8.1117. Retrieved from: www.researchgate.net

Galante, J., Dufour, G., Vainre,M., Wagner, P. A., Stochl, J., Benton, A., Lathia, N., Howarth, E., Jones, B. P. (2018). A mindfulness-based intervention to increase resilience to stress in university students (the Mindful Student Study): a pragmatic randomized controlled trial. Published in: The Lancet public Health, Volume 3, Issue 2, February 2018, Pages e72-e81. Retrieved from: www.sciencedirect.com

Coulon, M. S., Monroe, M. C., West, S. D. ( 2016). A Systematic, Multi-domain Review of Mobile Smartphone Apps for Evidence-Based Stress Management. Published in: American Journal of Preventive Medicine, Volume 51, Issue 1, July 2016, Pages 95-105. Retrieved from: www.sciencedirect.com
Chittaro, L., Sioni, R. (2014). Evaluating mobile apps for breathing training: The effectiveness of visualization. Published in: Computers in Human Behavior, Volume 40, November 2014, Pages 56-63. Retrieved from: www.sciencedirect.com

Ahtinen, A., Mattila, E., Välkkynen, P., Kaipainen, K., Vanhala, T., Ermes, M., Sairanen, E., Myllymäki, T., \& Lappalainen, R. (2013). Mobile Mental Wellness Training for Stress Management: Feasibility and Design Implications Based on a One-Month Field Study. Published in JMIR Mhealth and Uhealth. 2013 Jul-Dec; 1(2): e11, PMCID: PMC4114468. Published online 2013 Jul 10. doi: 10.2196/mhealth.2596, PMID: 25100683 Retrieved from: www.ncbi.nlm.nih.gov

Martín, B., De La Torre, Zapirain, G., Coronado, L., Rodrigues, J. (2018). Managing and Controlling Stress Using mHealth: Systematic Search in App Stores. Published in: JMIR Mhealth and Uhealth. 2018 May 9;6(5):e111. doi: 10.2196/mhealth.8866. Retrieved from: www.ncbi.nlm.nih.gov

Bakker, D., Rickard, N. (2018). Engagement in mobile phone app for self-monitoring of emotional wellbeing predicts changes in mental health: MoodPrism. Published in: Journal of Affective Disorders, Volume 227, February 2018, Pages 432-442, Retrieved from: www.sciencedirect.com

Christmann, A. C., Hoffmann, A. \& Bleser, G. (2017). Stress Management Apps With Regard to EmotionFocused Coping and Behavior Change Techniques: A Content Analysis. Published in: JMIR Mhealth Uhealth. 2017 Feb; 5(2): e22. PMCID: PMC5344985, Published online 2017 Feb 23. doi:10.2196/mhealth.6471 PMID: 28232299 Retrieved from: www.ncbi.nlm.nih.gov

(2017). The Stressless Mobile App Study: Helping carers thrive. This is a publication of: The School of Psychology, Deakin University The Australian Centre on Quality of Life \& Deakin University Australian Unity. Published by Deakin University, Geelong, Victoria 3217, Australia. ISBN Number: 978-0-7300-0020-4

Reid, S. C., Kauer, S. D., Hearps, S. J., Crooke, A. H., Khor, A.S., Sanci, L. A., Patton, G. C. (2011). A mobile phone application for the assessment and management of youth mental health problems in primary care: a randomised controlled trial. Published in: BMC Family Practice. 2011; 12: 131, PMCID: PMC3247177. Published online: 2011 Nov 29. doi: 10.1186/1471-2296-12-131, PMID: 22123031. Retrieved from: www.ncbi.nlm.nih.gov

Morrison, G. L., Geraghty, W.A. A., Lloyd, S., Goodman, N., Michaelides, T. D. Hargood, C., Weal, M., Yardley, L. (2018). Comparing usage of a web and app stress management intervention: An observational study. Published in: Internet Interventions Volume 12, June 2018, Pages 74-82. Retrieved from: www.sciencedirect.com

Kaipainen, K. (2014). Design and evaluation of online and mobile applications for stress management and healthy eating. ISBN 978-951-38-8124-5(URL: http://www.vtt.fi/publications/index.jsp). Pages: 23-24 \& 34-36

Grossmana, P., Niemannb, L., Schmidt, S., Walach, H. (2003). Mindfulness-based stress reduction and health benefits A meta-analysis. Published in: Journal of 
Psychosomatic Research 57 (2004) 35 - 43, Retrieved from: www.sciencedirect.com (Book style) 